\title{
Antifungal drug resistance: an update
}

\author{
David W Denning
}

\section{Correspondence to}

Professor David W Denning, The University of Manchester, Manchester M13 9PL, UK; ddenning@manchester.ac.uk

Received 12 March 2021 Accepted 6 November 2021

EAHP Statement 6: Education and Research.
Check for updates

(C) European Association of Hospital Pharmacists 2022. No commercial re-use. See rights and permissions. Published by BMJ.

To cite: Denning DW.

Eur J Hosp Pharm

2022;29:109-112.

\section{ABSTRACT}

The number of antifungal classes is small, and resistance is becoming a much more frequent problem. Much greater emphasis needs to be placed on susceptibility testing and antifungal stewardship. Such efforts demonstrably improve survival and overall clinical outcomes. Positively diagnosing a fungal infection with laboratory markers often allows antibacterial therapy to be stopped (ie, anti-tuberculous therapy in chronic pulmonary aspergillosis or antibiotics other than cotrimoxazole in Pneumocystis pneumonia), contributing to antimicrobial resistance control generally. Non-culture based diagnostics for fungal disease are transformational in terms of sensitivity and speed, but only occasionally identify antifungal resistance.

Systemic antifungal therapy is crucial for survival and reduction in morbidity of a wide range of fungal infections - some invasive (ie, invasive aspergillosis and candidaemia), some chronic (ie, chronic pulmonary aspergillosis and mycetoma), some allergic (ie, fungal asthma) and a very large number that are superficial (ie, oral or vaginal candidiasis, tinea capitis or corporis (ringworm)). The current systemic antifungal drugs included on the WHO Essential Medicines List are shown in table $1 .^{1}$

Antifungal resistance may be intrinsic or acquired. $^{2}$ Genus or species identification often reveals intrinsic resistance such as fluconazole resistance in Candida krusei, amphotericin B resistance in Aspergillus terreus or echinocandin resistance in Cryptococcus species. A tendency for higher rates of acquired resistance is also revealed by species identification, such as fluconazole resistance in Candida glabrata or azole resistance in Aspergillus fumigatus. So fungal identification is critical to good treatment decisions. A guideline on the therapy of rare mould infections has recently been published by the European Confederation of Medical Mycology which addressed therapy for intrinsically resistant mould fungi. ${ }^{3}$

However, the majority of antifungal resistance problems are acquired, meaning that the majority of strains from that species are susceptible, but some are not and have acquired resistance. The mechanisms of acquired resistance are many, and research continues to uncover more; many are combinations of changes leading to resistance. For azole resistance in Candida, the most common mechanisms are efflux (ie, increased export of drug from inside the fungal cell to the exterior), with less common causes related to chromosomal (or part chromosomal) duplication or target site mutation. ${ }^{4}$ In A. fumigatus, the most common cause is target site mutation, often combined with increased copy number of the target gene (CYP51A). ${ }^{5}$ Occasional strains of A. fumigatus have increased efflux of azoles, additional copies of a CYP51B protein and several other mechanisms conferring resistance. In Cryptococcus neoformans, chromosomal deletions (aneuploidy or disomy) account for most fluconazole resistance. ${ }^{67}$ Different mechanisms account for amphotericin B, flucytosine, echinocandin and terbinafine resistance.

\section{RESISTANCE IN DERMATOPHYTES}

Tinea capitis, cruris, corporis and pedis are common infections across the world, affecting $\sim 1$ billion people. They are caused by a variety of filamentous fungi including Trichophyton, Microsporum and Epidermophyton species. Tinea capitis is especially common in children in Africa, affecting an estimated 138 million. ${ }^{8}$ Microsporum canis infections are more difficult to treat and are refractory to terbinafine. ${ }^{9}$

In recent years, increasing resistance to terbinafine in Trichophyton interdigitale is increasingly recognised, especially in India. Multiple strains of T. interdigitale causing tinea corporis or tinea cruris are terbinafine resistant. For example, Khurana and colleagues analysed 64 strains from patients and 39 (61\%) had elevated minimum inhibitory concentrations (MICs) to terbinafine (MIC $>1 \mathrm{mg} / \mathrm{L}) .^{10}$ The European Committee on Antimicrobial Susceptibility Testing Antifungal Susceptibility Testing Subcommittee (EUCAST AFST) has determined an epidemiological MIC cut-off (ECOFF) of $0.125 \mathrm{mg} / \mathrm{L}$ for $T$. interdigitale and $0.03 \mathrm{mg} / \mathrm{L}$ for Trichophyton rubrum. ${ }^{11}$ Common substitutions found in the target gene squalene epoxidase (ErgA) were usually associated with MICs of $>32 \mathrm{mg} / \mathrm{L}$, with others at 4 and $8 \mathrm{mg} / \mathrm{L}$. All isolates were resistant to fluconazole in vitro but fortunately almost all are susceptible to itraconazole. Higher doses of itraconazole (ie, $400 \mathrm{mg}$ daily) give response rates of $\sim 65 \%$. The recent emergence of resistance is notionally attributed to over the counter medication, incomplete courses of therapy and combined steroid, antifungal and antibacterial creams with incomplete coverage and low exposures. ${ }^{12}$ The spread of resistance to other countries is documented. This resistant grouping of dermatophytes have been renamed as Trichophyton indotineae. ${ }^{13}$

Susceptibility testing of dermatophyte fungi is not usually done in Europe, but is now necessary in patients who are unresponsive to terbinafine. Multicentre validated methodology studies have been conducted by the EUCAST group. ${ }^{14}$ National surveillance for dermatophyte resistance is required in countries with extensive links to the Indian subcontinent, probably using a sentinel site approach, preferably including clinical outcome data. 
Table 1 Essential antifungal agents as assessed by the WHO. Access and antifungal price by country is visible here: https://wwwgaffiorg/ antifungal-drug-maps/.

\begin{tabular}{|c|c|c|c|}
\hline Antifungal & Route(s) & Primary indications & Resistance concerns \\
\hline Griseofulvin & Oral & Tinea corporis and capitis & Some clinical resistance described \\
\hline Fluconazole & Oral, IV & $\begin{array}{l}\text { Mucosal candidiasis, prophylaxis in leukaemia, HSCT and } \\
\text { intensive care, treatment and maintenance therapy for } \\
\text { cryptococosis }\end{array}$ & $\begin{array}{l}\text { All moulds, including Aspergillus resistant. Lower response rates for } \\
\text { endemic mycoses such as histoplasmosis. All Candida auris and Candida } \\
\text { krusei strains resistant-some other species less susceptible or resistant }\end{array}$ \\
\hline Amphotericin B & $\begin{array}{l}\text { IV and } \\
\text { topical }\end{array}$ & $\begin{array}{l}\text { Invasive candidiasis and cryptococcal meningitis, endemic } \\
\text { fungal infections. Empiric therapy in febrile neutropenia. } \\
\text { Lower response rate for invasive aspergillosis than azoles }\end{array}$ & $\begin{array}{l}\text { Aspergillus terreus and nidulans resistant. Some strains of Candida auris } \\
\text { resistant. Several intrinsically resistant fungi }\end{array}$ \\
\hline Flucytosine & Oral, IV* & $\begin{array}{l}\text { Cryptococcal meningitis, neonatal candidiasis and Candida } \\
\text { endocarditis and endophthalmitis, other rare fungal } \\
\text { infections }\end{array}$ & $\begin{array}{l}\text { Low levels of resistance in Candida and Cryptococcus. Aspergilli and } \\
\text { most moulds and endemic fungi resistant }\end{array}$ \\
\hline Itraconazole & Oral, IV* & $\begin{array}{l}\text { All skin infections, all forms of aspergillosis, endemic fungal } \\
\text { infections, mucosal candidiasis, prophylaxis in leukaemia }\end{array}$ & $\begin{array}{l}\text { Rising problems with resistance in Aspergillus fumigatus, flavus and niger. } \\
\text { Some cross resistance with fluconazole in Candida }\end{array}$ \\
\hline Voriconazole & Oral, IV & Invasive and chronic aspergillosis, some rare moulds & Some azole cross resistance in Aspergillus. Mucorales intrinsically resistant \\
\hline Natamycin $5 \%$ & $\begin{array}{l}\text { Topical, } \\
\text { eye }\end{array}$ & Fungal keratitis & Most effective agent, but some rarer fungi resistant, probably \\
\hline $\begin{array}{l}\text { Echinocandins } \\
\text { (micafungin, caspofungin, } \\
\text { anidulafungin) }\end{array}$ & IV & $\begin{array}{l}\text { Candidaemia, invasive candiasis, invasive and chronic } \\
\text { pulmonary aspergillosis, prophylaxis }\end{array}$ & $\begin{array}{l}\text { Most effective agent for most Candida infections, notably the majority of } \\
\text { Candida auris strains. Less effective than azoles for aspergillosis. }\end{array}$ \\
\hline
\end{tabular}

anidulafungin)

*Many countries only have oral

HSCT, haematopoetic stem cell transplant; ; IV, intravenous.

\section{TRIAZOLE RESISTANCE IN ASPERGILLUS FUMIGATUS}

A. fumigatus is the most common cause globally of invasive, chronic and allergic aspergillosis, collectively affecting $\sim 10$ million people. The only oral class of antifungal agents active against Aspergillus species is the triazole groupitraconazole and voriconazole (both WHO Essential Medicines) and posaconazole and isavuconazole. In 2007, alarming reports from Manchester and Nijmegen described increasing azole resistance in A. fumigatus. ${ }^{15}$ Broadly, two circumstances led to the growing resistance problem. First, in the environment strains highly resistant to azole and triazoles emerged rapidly as a consequence of the widespread use of azole fungicides (about 1/3 of all fungicide use). Such strains are characterised by two principal genetic signatures $\left(\mathrm{TR}_{34} / \mathrm{L} 98 \mathrm{H}\right.$ and $\mathrm{TR}_{46} / \mathrm{Y} 121 \mathrm{~F} /$ $\mathrm{T} 289 \mathrm{~A}) .^{5}{ }^{16}$ Second, in patients on long-term therapy, strains acquire a variety of resistance mechanisms including target site mutations, increased target copy number, efflux and other mechanisms still being described. ${ }^{17} 18$ Most isolates that are resistant are resistant to at least two triazoles and most are pan-azole resistant. Resistance has been seen in every continent except Antarctica. Resistant rates in Europe vary from 1-20\%, with higher rates in northern Europe. ${ }^{19}$ In Yunnan province in China, cultures from greenhouses found $\sim 80 \%$ of $A$. fumigatus resistant to at least one medical triazole drug, with $>30 \%$ showing crossresistance to both itraconazole and voriconazole. ${ }^{20}$ In southern Vietnam, azole resistance rates in A. fumigatus are about $90 \% .^{21}$ Strains of Aspergillus flavus may also be azole resistant, with a recent report from Vietnam finding $\sim 50 \%$ of environmental strains to be multi-azole resistant and $85 \%$ resistant to itraconazole. $^{22}$ These were linked to aquaculture, a new association.

Susceptibility testing of Aspergillus species is now well established and available in most developed countries. Aspergillus niger strains are always resistant to itraconazole and isavuconazole, and Aspergillus terreus and nidulans to amphotericin B. It is generally recommended to susceptibility test all strains grown in patients taking antifungal therapy (not including fluconazole), as resistance is suggested by a positive culture on therapy, and preferably all strains in whom therapy is planned, whether from invasive, chronic or allergic aspergillosis. ${ }^{23}$ While susceptibility testing can be slow, direct PCR detection of resistance is possible, particularly for the mutations found in the environment. ${ }^{24-26}$ These data can all be part of antifungal stewardship which needs more emphasis in hospital practice. It would be helpful if the antifungal resistance programme run by the WHO Global Antimicrobial Resistance Surveillance System (GLASS) included surveillance for itraconazole and voriconazole resistance in Aspergillus. ${ }^{27}$

Patients with triazole resistance fail therapy. ${ }^{17} \mathrm{~A}$ recent cohort study showed that the mortality in voriconazole-resistant invasive aspergillosis was 20-30\% higher than in patients with voriconazole-susceptible disease, ${ }^{16}$ indicating that the major advances in survival of patients through azole-based therapy are completely lost in resistant cases.

Alternative treatments for pan-azole resistance are all intravenous-amphotericin B and either micafungin or caspofungin (no data for anidulafungin). These agents are 15-20\%less effective than azoles for invasive aspergillosis and are troublesome to administer long term. Some data are published on long term usage for chronic pulmonary aspergillosis. ${ }^{28} 29$ There are several new agents in clinical development for aspergillosis that may address this problem, including rezafungin (once weekly IV), ${ }^{30}$ ibrexafungerp (oral), ${ }^{31}$ olorofim (IV and oral) ${ }^{32}$ and fosmanogepix (IV and oral). ${ }^{33}$

To help retain the medically important azoles, reduction of usage of these triazole fungicides in the environment is required. ${ }^{18}$ This could be a voluntary withdrawal from some or all fungicide market segments. New antifungal drugs with novel chemistries and modes of action in clinical development and/or commercially launched after regulatory approval should never be used as fungicides in agriculture. The authorisation procedure for new fungicides should include testing for activity against non-target fungi such as A. fumigatus, that are known to cause infections in humans.

\section{MULTI-DRUG RESISTANT CANDIDA GLABRATA AND CANDIDA AURIS}

The optimal therapy of invasive candidiasis and candidaemia relies on an intravenous echinocandin (caspofungin, micafungin 
and andiulafungin) with the exception of a less susceptible or resistance species such as Candida parapsilosis complex, Candida guilliermondii (rare) and Candida famata (rare). In these cases amphotericin B or fluconazole are used, occasionally augmented with flucytosine combination therapy. Unfortunately C. glabrata is poorly responsive to fluconazole and is often associated with urinary tract infection (which is itself very common in hospitalised, catheterised patients). Echinocandin therapy is usually successful for systemic C. glabrata infection, but none of the echinocandin drugs are excreted into the urine. Some resistance to fluconazole is reported in Candida albicans, Candida tropicalis and C. parapsilosis, but this is not addressed here in detail.

What has emerged in the last 5 years has been multi-drug resistance in C. glabrata and global spread of Candida auris. In the 2015 Asia study of candidaemia, C. glabrata was the causative species in $14 \%$ of cases, ${ }^{34}$ and in a later study of intensive care unit candidaemia in India ( $\mathrm{n}=918$ strains), C. auris caused 8.2\% and C. glabrata 7.1\%. ${ }^{35}$ Multi-drug resistant (MDR) Candida species were found in $1.9 \%$.

Increasing rates of MDR C. glabrata have been documented in candidaemia studies comparing rates over time. The number of cases is probably underestimated, as only initial blood isolates are included in surveillance studies. One study demonstrated echinocandin resistance in $21.6 \%$ of C. glabrata isolates from patients exposed to echinocandins for 7 days or longer. ${ }^{36}$ MDR C. glabrata substantially increases mortality.

Resistant C. glabrata are also a significant issue for women with recurrent vulvovaginal candidiasis (rVVC) as intravenous therapy is inappropriate for these women and many become untreatable. At any one time, an estimated 138 million women suffer from rVVC, ${ }^{37}$ and repetitive courses of local azoles (ie, clotrimazole) or oral fluconazole, almost certainly are directly linked to replacement of C. albicans with C. glabrata.

C. auris has caused outbreaks across the world and is now endemic in many countries including the USA (ie, New York), ${ }^{38}$ South Africa, Colombia and India, ${ }^{39} 40$ to name a few examples. One outbreak occurred in a neonatal unit in Colombia. ${ }^{41}$

The vast majority of clinical strains of $C$. auris are resistant to fluconazole, and the proportion that are also echinocandin or amphotericin B resistant varies by study. A common mechanism of fluconazole resistance is one of several mutations in zinc-cluster transcription factor-encoding gene TAC1B which increases drug efflux via increased CDR1 expression. ${ }^{42}$ Almost all are susceptible to flucytosine but resistance emerges rapidly on therapy. A small proportion (3-10\%) are pan-resistant and currently untreatable. Echinocandin prophylaxis is ineffective as it does not penetrate adequately to the skin surface where C. auris resides, and so breakthroughs of MDR C. auris are promoted by this practice.

Fluconazole resistant C. parapsilosis is also a problem and can cause outbreaks. One large study found widespread dissemination in South Africa, ${ }^{43}$ especially in private hospitals, and other outbreaks have been described in Turkey and Mexico. ${ }^{44} 45$

All blood and other sterile site cultures of Candida should be identified to species level and susceptibility tested. Stewardship programmes should focus in part on stopping unnecessary antifungal therapy for suspected cases of candidiasis and for a positive culture which is not significant (notably respiratory samples). The use of rapid beta 1,3-D-glucan testing can be useful to allow the cessation of therapy as it has a high negative predictive value. ${ }^{46}$

\section{ANTIFUNGAL STEWARDSHIP}

Several studies have convincingly shown that antifungal stewardship reduces mortality in the hospitalised patient. ${ }^{47-52}$ The basic elements of successful stewardship are: (1) comprehensive knowledge and continuous reference to the best clinical guidance on fungal disease management; (2) a primary focus on the best quality care, not on cost saving, as some expensive antifungals are the best choice (stopping unnecessary therapy is what saves money); (3) clinical experience to know when to infer likely results if not yet available or samples cannot or were not taken. One important tool to assist in top quality fungal disease stewardship is rapid diagnostic services, notably mycology results, but also imaging. Too often, turnaround time for the results takes days, and so empirical (and often wrong) choices need to be made. Another key tool is antifungal therapeutic drug monitoring (TDM), especially for voriconazole and itraconazole, but also flucytosine in neonates and patients with renal dysfunction. ${ }^{53} \mathrm{~A}$ third key tool is immediate access to drug interaction data-one online tool which is curated weekly provides this, and can be downloaded onto Android phones. ${ }^{54}$

Outpatient antifungal stewardship relies on experienced clinicians seeing complex patients, and not assuming that 'any old clinic' will do. If long-term antifungal therapy is started, the follow-up requires those patients to see experienced clinicians for optimal care, including TDM if appropriate.

In many situations, if a fungal disease is diagnosed then antibacterial therapy can be stopped or drastically reduced..$^{55}$ Good examples are a diagnosis of chronic pulmonary aspergillosis so that tuberculosis can be ruled out, Pneumocystis pneumonia when only cotrimoxazole is needed, candidaemia, fungal asthma and Aspergillus bronchitis when treatment with antifungals drastically reduces exacerbations and antibacterial prescriptions.

Funding The authors have not declared a specific grant for this research from any funding agency in the public, commercial or not-for-profit sectors.

Competing interests The author and his family hold Founder shares in F2G Ltd, a University of Manchester spin-out antifungal discovery company. He acts or has recently acted as a consultant to Pulmatrix, Pulmocide, Zambon, Biosergen, Bright Angel Therapeutics, Cipla and Metis. He sits on the DSMB for a SARS CoV2 vaccine trial. In the last 3 years, he has been paid for talks on behalf of Dynamiker, Hikma, Gilead, Merck, Mylan and Pfizer. He is a longstanding member of the Infectious Disease Society of America Aspergillosis Guidelines group, the European Society for Clinical Microbiology and Infectious Diseases Aspergillosis Guidelines group.

Patient consent for publication Not applicable.

Provenance and peer review Commissioned; externally peer reviewed.

Data availability statement No data are available. No specific new data.

ORCID iD

David W Denning http://orcid.org/0000-0001-5626-2251

\section{REFERENCES}

1 World Health Organization. The selection and use of essential in vitro diagnostics TRS 1031. WHO Technical Report Series, No. 1031, 202129 January, 2021. Available: https://www.who.int/publications/i/item/9789240019102 [Accessed 12 Mar 2021].

2 Arastehfar A, Gabaldón T, Garcia-Rubio R, et al. Drug-resistant fungi: an emerging challenge threatening our limited antifungal armamentarium. Antibiotics 2020;9:877.

3 Hoenigl M, Salmanton-García J, Walsh TJ, et al. Global guideline for the diagnosis and management of rare mould infections: an initiative of the European Confederation of Medical Mycology in cooperation with the International Society for Human and Animal Mycology and the American Society for Microbiology. Lancet Infect Dis 2021;21:e246-57.

4 Bhattacharya S, Sae-Tia S, Fries BC. Candidiasis and mechanisms of antifungal resistance. Antibiotics 2020:9:312.

5 Nywening AV, Rybak JM, Rogers PD, et al. Mechanisms of triazole resistance in Aspergillus fumigatus. Environ Microbiol 2020;22:4934-52.

6 Stone NR, Rhodes J, Fisher MC, et al. Dynamic ploidy changes drive fluconazole resistance in human cryptococcal meningitis. J Clin Invest 2019;129:999-1014. 
7 Naicker SD, Mpembe RS, Maphanga TG, et al. Decreasing fluconazole susceptibility of clinical South African Cryptococcus neoformans isolates over a decade. PLoS Negl Trop Dis 2020;14:e0008137.

8 Bongomin F, Olum R, Nsenga L, et al. Burden of tinea capitis among children in Africa: protocol for a systematic review and meta-analysis of observational studies, 1990 2020. BMJ Open 2020;10:e041230.

9 Dragos V, Lunder M. Lack of efficacy of 6-week treatment with oral terbinafine for tinea capitis due to Microsporum canis in children. Pediatr Dermatol 1997;14:46-8.

10 Khurana A, Masih A, Chowdhary A, et al. Correlation of in vitro susceptibility based on MICs and squalene epoxidase mutations with clinical response to terbinafine in patients with tinea corporis/cruris. Antimicrob Agents Chemother 2018;62:e01038-18.

11 EUCAST AFST. Overview of antifungal ECOFFs and clinical breakpoints for yeasts, moulds and dermatophytes using the EUCAST E.Def 7.3, E.Def 9.3 and E.Def 11.0 procedures. Available: https://www.eucast.org/astoffungi/clinicalbreakpointsforantifu ngals/[Accessed 22 Apr 2021].

12 Verma SB. Topical corticosteroid misuse in India is harmful and out of control. BM 2015;351:h6079.

13 Kano R, Kimura U, Kakurai M, et al. Trichophyton indotineae sp. nov:: a new highly terbinafine-resistant anthropophilic dermatophyte species. Mycopathologia 2020;185:947-58

14 Arendrup MC, Jørgensen KM, Guinea J, et al. Multicentre validation of a EUCAST method for the antifungal susceptibility testing of microconidia-forming dermatophytes. J Antimicrob Chemother 2020;75:1807-19.

15 Verweij PE, Howard SJ, Melchers WJG, et al. Azole-resistance in Aspergillus: proposed nomenclature and breakpoints. Drug Resist Updat 2009:12:141-7.

16 Lestrade PPA, Buil JB, van der Beek MT, et al. Paradoxal trends in azole-resistant Aspergillus fumigatus in a national multicenter surveillance program, the Netherlands, 2013-2018. Emerg Infect Dis 2020;26:1447-55.

17 Howard SJ, Cerar D, Anderson MJ, et al. Frequency and evolution of azole resistance in Aspergillus fumigatus associated with treatment failure. Emerg Infect Dis 2009;15:1068-76.

18 Bowyer P, Bromley MJ, Denning DW. Linking calcium signaling and mitochondria function in fungal drug resistance. Proc Natl Acad Sci U S A 2020;117:1254-6.

19 Verweij PE, Lucas JA, Arendrup MC, et al. The one health problem of azole resistance in Aspergillus fumigatus: current insights and future research agenda. Fungal Biol Rev 2020;34:202-14.

20 Zhou D, Korfanty GA, Mo M, et al. Extensive genetic diversity and widespread azole resistance in greenhouse populations of Aspergillus fumigatus in Yunnan, China. mSphere 2021;6:e00066-21.

21 Duong T-MN, Le T-V, Tran K-LH, et al. Azole-resistant Aspergillus fumigatus is highly prevalent in the environment of Vietnam, with marked variability by land use type. Environ Microbio/ 2021. doi:10.1111/1462-2920.15660. [Epub ahead of print: $07 \mathrm{Jul}$ 2021].

22 Duong TMN, Nguyen PT, Le TV, et al. Drug-resistant Aspergillus flavus is highly prevalent in the environment of Vietnam: a new challenge for the management of aspergillosis? J Fungi 2020;6:296.

23 Schelenz S, Barnes RA, Barton RC. British Society for Medical Mycology: diagnostic standards for the management of serious fungal diseases. Lancet Infect Dis 2015:15:461-74.

24 Pelzer BW, Seufert R, Koldehoff M, et al. Performance of the AsperGenius ${ }^{\circledR}$ PCR assay for detecting azole resistant Aspergillus fumigatus in BAL fluids from allogeneic HSCT recipients: a prospective cohort study from Essen, West Germany. Med Mycol 2020:58:268-71.

25 Wang Q, Kontoyiannis DP, Li R, et al. A novel broad allele-specific TaqMan real-time PCR method to detect triazole-resistant strains of Aspergillus fumigatus, even with a very low percentage of triazole-resistant cells mixed with triazole-susceptible cells. J Clin Microbiol 2019;57:e00604-19.

26 Novak-Frazer L, Anees-Hill SP, Hassan D. Deciphering Aspergillus fumigatus triazole resistance in situ in respiratory specimens via pyrosequencing of cyp51A. J Antimicrob Chemother 2020;75:3501-9.

27 World Health Organisation. Global antimicrobial resistance surveillance system (GLASS). Available: https://www.who.int/glass/en/ [Accessed 22 Apr 2021].

28 Otu AA, Bongomin F, Bazaz R, et al. Micafungin may be safely administered as outpatient parenteral antimicrobial therapy for chronic pulmonary aspergillosis. Mycoses 2019;62:152-6.

29 Newton PJ, Harris C, Denning DW. Impact of AmBisome therapy on chronic pulmonary aspergillosis. J Infect 2016:73:485-95.

30 Garcia-Effron G. Rezafungin-mechanisms of action, susceptibility and resistance: similarities and differences with the other echinocandins. J Fungi 2020:6:262.
31 Jallow S, Govender NP. Ibrexafungerp: a first-in-class oral triterpenoid glucan synthase inhibitor. J Fungi 2021;7:163.

32 Wiederhold NP. Review of the novel investigational antifungal Olorofim. J Fungi 2020;6:122.

33 Shaw KJ, Ibrahim AS. Fosmanogepix: a review of the first-in-class broad spectrum agent for the treatment of invasive fungal infections. J Fungi 2020;6:239.

34 Tan BH, Chakrabarti A, Li RY, et al. Incidence and species distribution of candidaemia in Asia: a laboratory-based surveillance study. Clin Microbiol Infect 2015;21:946-53.

35 Chakrabarti A, Sood P, Rudramurthy SM, et al. Incidence, characteristics and outcome of ICU-acquired candidemia in India. Intensive Care Med 2015:41:285-95.

36 Jensen RH, Johansen HK, Søes LM, et al. Posttreatment antifungal resistance among colonizing Candida isolates in candidemia patients: results from a systematic multicenter study. Antimicrob Agents Chemother 2015;60:1500-8.

37 Denning DW, Kneale M, Sobel JD, et al. Global burden of recurrent vulvovaginal candidiasis: a systematic review. Lancet Infect Dis 2018;18:e339-47.

38 Zhu Y, O'Brien B, Leach L, et al. Laboratory analysis of an outbreak of Candida auris in New York from 2016 to 2018: impact and lessons learned. J Clin Microbiol 2020;58:e01503-19.

39 van Schalkwyk E, Mpembe RS, Thomas J, et al. Epidemiologic shift in candidemia driven by Candida auris, South Africa, 2016-2017'. Emerg Infect Dis 2019:25:1698-707.

40 Chowdhary A, Tarai B, Singh A, et al. Multidrug-resistant Candida auris infections in critically ill coronavirus disease patients, India, April-July 2020. Emerg Infect Dis 2020;26:2694-6

41 Alvarado-Socarras JL, Vargas-Soler JA, Franco-Paredes C, et al. A cluster of neonatal infections caused by Candida auris at a large referral center in Colombia. J Pediatric Infect Dis Soc 2021;10:549-55.

42 Rybak JM, Muñoz JF, Barker KS, et al. Mutations in TAC1B: a novel genetic determinant of clinical fluconazole resistance in Candida auris. mBio 2020;11:e00365-20.

43 Govender NP, Patel J, Magobo RE, et al. Emergence of azole-resistant Candida parapsilosis causing bloodstream infection: results from laboratory-based sentinel surveillance in South Africa. J Antimicrob Chemother 2016;71:1994-2004.

44 Arastehfar A, Daneshnia F, Hilmioğlu-Polat $\mathrm{S}$, et al. First report of candidemia clonal outbreak caused by emerging fluconazole-resistant Candida parapsilosis isolates harboring Y132F and/or Y132F+K143R in Turkey. Antimicrob Agents Chemother 2020;64:e01001-20

45 Corzo-Leon DE, Peacock M, Rodriguez-Zulueta P, et al. General hospital outbreak of invasive candidiasis due to azole-resistant Candida parapsilosis associated with an Erg11 Y132F mutation. Med Mycol 2021;59:664-71.

46 Rautemaa-Richardson R, Rautemaa V, Al-Wathiqi F, et al. Impact of a diagnosticsdriven antifungal stewardship programme in a UK tertiary referral teaching hospital. $J$ Antimicrob Chemother 2018:73:3488-95.

47 Machado M, Chamorro de Vega E, Martínez-Jiménez MdelC, et al. Utility of 1,3 $\beta$-Dglucan assay for guidance in antifungal stewardship programs for oncologic patients and solid organ transplant recipients. J Fungi 2021;7:59.

48 Vena A, Bouza E, Corisco R, et al. Efficacy of a "checklist" intervention bundle on the clinical outcome of patients with candida bloodstream infections: a quasiexperimental pre-post study. Infect Dis Ther 2020;9:119-35.

49 Martín-Gutiérrez G, Peñalva G, Ruiz-Pérez de Pipaón M, et al. Efficacy and safety of a comprehensive educational antimicrobial stewardship program focused on antifungal use. J Infect 2020;80:342-9.

50 Hart $E$, Nguyen $M$, Allen $M$, et al. A systematic review of the impact of antifungal stewardship interventions in the United States. Ann Clin Microbiol Antimicrob 2019;18:24

51 Benoist H, Rodier S, de La Blanchardière A, et al. Appropriate use of antifungals: impact of an antifungal stewardship program on the clinical outcome of candidaemia in a French university hospital. Infection 2019:47:435-40.

52 Kawaguchi H, Yamada K, Imoto W, et al. The effects of antifungal stewardship programs at a tertiary-care teaching hospital in Japan. J Infect Chemother 2019;25:458-62.

53 Ashbee HR, Barnes RA, Johnson EM, et al. Therapeutic drug monitoring (TDM) of antifungal agents: guidelines from the British Society for Medical Mycology. J Antimicrob Chemother 2014;69:1162-76.

54 Antifungal interactions database and smartphone APPs. Available: https://www. aspergillus.org.uk/antifungal-drug-interactions/ [Accessed 22 Apr 2021]

55 Denning DW, Perlin DS, Muldoon EG, et al. Delivering on antimicrobial resistance agenda not possible without improving fungal diagnostic capabilities. Emerg Infect Dis 2017;23:177-83. 